\section{New technology coming soon}

Later this year, Align Technology will introduce several enhancements to improve Invisalign Go and will offer a new Services Scheme to improve dentists' clinical and practice experience. In

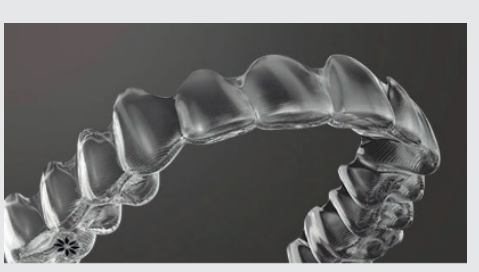

July 2018, the company will be launching a new,

improved Invisalign Go with a more user-friendly digital platform and a product that offers more flexible and expanded treatment options.

Invisalign Go was designed to help general practitioners treat their patients more successfully and help them achieve a new smile; its expanded tooth movement capabilities and flexibility will enable them to treat wider range of cases:

1. Treat 5-5 (2nd pre-molar to 2 nd pre-molar) for greater arch expansion, and ability to treat more mild to moderate crowding cases

2. Increased vertical movements for levelling incisors and anterior teeth

3. Support improved patient uptake by comparing multiple treatment plans

4. Increased number of aligners to help tailor treatment plans for your patients' diverse set of needs (up to 20 aligners).

Align Technology aims to provide dental professionals with cutting-edge solutions which help grow practices and enable patients achieve new, beautiful smiles using clear aligner therapy. General practitioners keen to find out more about Invisalign Go can visit www. Invisalign.co.uk or speak with their Invisalign Go customer care representative.

\section{Quick to impress}

Recognised for its commitment to innovation and constant product development, 3M Oral Care did not disappoint at the British Dental Conference and Dentistry Show 2018.

The team revealed the new and improved Impregum Super Quick polyether impression material, offering the precision of a polyether alongside the speed of a VPS material. Its setting time of just two minutes certainly impressed visitors to the stand.

Other highlights included the highly aesthetic Filtek One bulk fill restorative, the strong RelyX Ultimate adhesive resin, the easy-to-handle Ketac Universal glass ionomer restorative and the time-saving Lava Esthetic fluorescent full-contour zirconia.

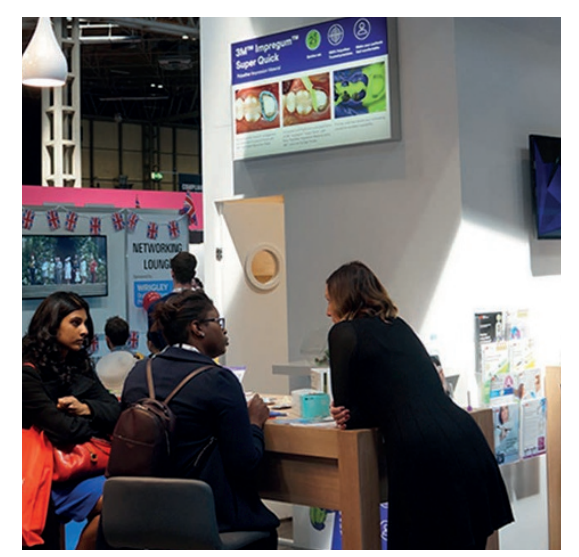

If you missed the team at the show, call today to find out more.

For more information, call 0800626578 or visit www.3M.co.uk/Dental.

\section{Inspiring Britain}

Rodericks Dental has been recognised in the fifth edition of the London Stock Exchange Group's (LSEG) 1000 Companies to Inspire Britain report. The full list represents a unique and diverse selection of businesses

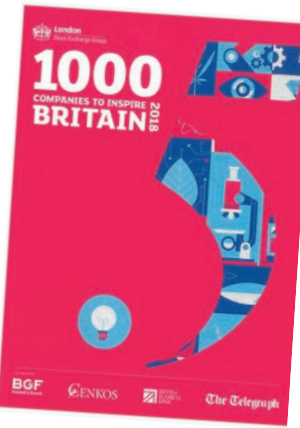

from across the UK that have not only demonstrated positive revenue growth over the past three years, but also outperformed others in their sectors.

With the support of the government and major political parties, the LSEG 1000 Companies to Inspire Britain report also considers the opportunities and challenged facing SMEs and evaluates sector trends.

This is the second time Rodericks has celebrated inclusion within the top 1,000 UK companies, having been recognised back in 2013.

Shalin Mehra, Chief Executive Officer Rodericks Dental, commented: 'Rodericks is first and foremost committed to excellent patient care. We are a clinically led group and we strive to offer various learning and career development opportunities for all our professionals. It is only with the dedication of our teams that we have been able to continue growing the business and increase access to dentistry across England and Wales. We are delighted to be recognised in the LSEG 1000 Companies to Inspire Britain report once again and I'd like to thank everyone who made this possible!.

For more information about Rodericks, visit www.rodericksdental.co.uk.

\title{
Ultra-short implant for reduced bone volume
}

The SKY implant family from bredent medical has been expanded with the new copaSKY - an ultra-short implant with a length of $5.2 \mathrm{~mm}$ and a diameter of 4.0 , 5.0 or $6.0 \mathrm{~mm}$.

copaSKY has the tried and tested osseo-connect-surface, which has enabled SKY implants to set milestones in recent decades in immediate restoration. The thread design and the special surface finish of the SKY implants guarantee high primary stability and rapid osseointegration. The conical parallel walled connection on the copaSKY is reversible and can be calculated in terms of height to avoid a complicated Morse taper effect.

With the copaSKY, bredent medical now offers the ideal solution for short, wide alveolar ridges. The available bone is used optimally and so time-consuming augmentations can be avoided.

The SKY family's proven surgical and prosthetic protocol also applies for the copaSKY, meaning that dentists can easily integrate the indication into their clinical workflows.

For more information about the new copaSKY, visit www.bredent.co.uk/ copaSKY or call 01246559599. 